$\$$ sciendo

\title{
BOLETIM IBÉRICO DE FENOMENOLOGIA
}

\author{
$2003-2004$
}

\section{BOLETÍN IBÉRICO DE FENOMENOLOGÍA}

$$
2003-2004
$$

María da Luz Pintos Peñaranda

Pedro M. S. Alves

Renato Epifânio 


\section{3}

\section{Monografias e artigos / Monografías y artículos}

1. ADRIÁN ESCUDERO, Jesús - "Vida y conciencia histórica en el joven Heidegger". In Jesús Díaz / Ma Carmen López Saénz (eds.) Fenomenología e Historia. Madrid: U.N.E.D., 2003, pp. 61-65.

2. ALVES, Pedro M. S. - Subjectividade e Tempo. Lisboa: CFUL, 2003, $445 \mathrm{pp}$.

3. ALVES, Pedro M. S. - "Uma introdução husserliana na filosofia da linguagem e da comunicação", Análise, Lisboa, 25 (2003), pp. 19-62.

4. ALVES, Pedro M. S. - "Fenomenologia del Lenguage y Comunicación: Una introducción husserliana". Escritos de Filosofia, 43 (2003), pp.183-227.

5. ARCE CARRASCOSO, José Luis - "La 'experiencia de la facticidad' en Heidegger, frente a la 'impresión de la realidad' en Zubiri". Convivium. Revista de Filosofia 16 (2003) 117-139.

6. ARIÑO VERDÚ, Amparo - "Historia e historicidad en el primer Sartre". In Jesús Díaz / Ma Carmen López Saénz (eds.) - Fenomenología e Historia. Madrid: U.N.E.D., 2003, pp. 111-126.

7. BARBOSA, Luís Marques - Ensaios sobre fenomenologia do conhecimento: do espelhamento à transcendência. Évora: Departamento de Pedagogia e Educação, 2003, 79 pp.

8. BECH, Joseph Maria - "El pensamiento de la no-coincidencia", Convivium. Revista de Filosofia, Barcelona, 16 (2003) 57-94.

9. BELO, Fernando - "O legado de Heidegger às ciências", Revista Portuguesa de Filosofia, LIX 4 (2003), pp. 1229-1239. 
10. BERNARDO, Fernanda - "Da Responsabilidade. Paradoxos: Derrida a ler Kierkegaard a ler O sacrificio de Isaac, Phainomenon, Lisboa, 5-6 (2002/2003), pp. 271-288.

11. BLANC, Mafalda de Faria - "Filosofia, Religião e Gnose", Actas do Colóquio A Mente, a Religião e a Ciência, Centro de Filosofia da Univ. de Lx., 2003, pp. 153-172.

12. BORGES-DUARTE, Irene - "Husserl e a fenomenologia heideggeriana da fenomenologia", Phainomenon, Lisboa, 7 (2003) pp. 87-104.

13. BORGES-DUARTE, Irene - "O melão, o remoinho e o tempo. Descartes e o sonho de uma noite de outono." Revista Portuguesa de Filosofia. Braga, 59 (2003), pp. 315-337.

14. CONILL, Jesús - "Pensar la vida desde la historia". In Diego Gracia (ed.), Ciencia y vida. Homenaje a Pedro Laín Entralgo. Bilbao: Fundación BBVA, 2003, pp. 107-133.

15. CORREIA, Carlos João - "Sentimento de si e subjectividade animal", in AA.VV., Ética Ambiental: uma Ética para o Futuro, coord. de Cristina Beckert, Lisboa, CFUL, 2003, pp. 73-86.

16. CORREIA, João Carlos - "Fenomenologia e Teoria dos Sistemas: reflexões sobre um encontro improvável", Revista Filosófica de Coimbra, vol. XII, nº 23 (2003), pp. 181-214.

17. COUTINHO, Jorge - "Hermenêutica e Verdade", Didaskalia, Lisboa, XXXIII/ 1-2 (2003), pp. 433-446.

18. DÍAZ ÁLVAREZ, Jesús M. - "Hacia la dimensión práctica de la fenomenología: Historia y filosofía de la historia en la fenomenología husserliana”. In Jesús Díaz / María del Carmen López Saénz (eds.) Fenomenología e Historia. Madrid: U.N.E.D., 2003, pp.35-47.

19. DÍAZ ÁlVAREZ, Jesús M. - Husserl y la historia. Hacia la función práctica de la fenomenología. Madrid: U.N.E.D., 2003. 
20. DOMINGO MORATALLA, Tomás - "Paul Ricoeur y la cuestión de la historia”. In Jesús Díaz / María del Carmen López Saénz (eds.) Fenomenología e Historia. Madrid: U.N.E.D., 2003, pp. 141-153.

21. DOMÍNGUEZ REY, Antonio - El drama del lenguaje. Madrid: Editorial Verbum / UNED, 2003.

22. DOMÍNGUEZ REY, Antonio - "Filosofía del lenguaje I. Fondo dicente del lenguaje II". In http://www.liceus.com

23. DOMÍNGUEZ REY, Antonio - "Ontopoética del significante: El palpo del signo”. Analecta Husserliana LXXIX (2003) 397-423.

24. DOMÍNGUEZ REY, Antonio - "Subsidio de la historia según E. Lévinas”. In Jesús Díaz / María del Carmen López Saénz (eds.) Fenomenología e Historia. Madrid: U.N.E.D., 2003, pp. 183-198.

25. FERNÁNDEZ BEITES, Pilar - "El cuerpo vivido y su identidad con el cuerpo objetivo". Escritos de Filosofia, Buenos Aires, 43 (2003) 229-258.

26. FERNÁNDEZ BEITES, Pilar - "Sustancia y temporalidad. Sobre la teoría scheleriana de la persona”, Revista de filosofia, México, 107 (2003), pp. 55-83.

27. FERRER SANTOS, Urbano - "Ampliaciones de la noción fenomenológica de mundo en L. Polo”, Studia Poliana 5 (2003) 263-280.

28. FERRER SANTOS, Urbano - Desarrollos de ética fenomenológica. Albacete: Moralea, 2003 ( $2^{a}$ edición ampliada).

29. FERRER SANTOS, Urbano - "Niveles de la vivencia temporal en Edith Stein”. In Jesús Díaz / Ma Carmen López Saénz (eds.) Fenomenología e Historia. Madrid: U.N.E.D., 2003, pp. 127-139.

30. GARCÍA-BARÓ, Miguel - "Esbozo de una estética", Arbor vol.CLXXV, 689 (2003) 629-647.

31. GIL, Fernando - A Convicção, trad. de Adelino Cardoso e Marta Lança, Porto, Campo das Letras, 2003, 240 pp. 
32. GÓMEZ ROMERO, Isidro - "La constitución del mundo objetivo". In José Manuel Santos, Pedro M. S. Alves, André Barata (eds.) - A Fenomenologia Hoje. Actas do primeiro Congresso da Associação Portuguesa de Filosofia Fenomenológica (Outubro de 2002). Lisboa: Centro de Filosofia da Universidade de Lisboa, 2003, pp. 125-135.

33. GONZÁLEZ LÓPEZ, José Luis - "La lectura husserliana de las Logische Untersuchungen desde el 'Vorrede' de 1913”. Agora. Papeles de Filosofia 22/1 (2003) 83-110.

34. GUTIÉRREZ POZO, Antonio - "Arte y Gelassenheit. Estética, ética y lógica originarias en el pensar de Heidegger”. Logos. Anales del Seminario de Metafisica 36 (2003) 153-186.

35. HELENO, José Manuel - Identidade Pessoal. Lisboa: Instituto Piaget, 2003.

36. HELENO, José Manuel - Fascinio e Perturbação. Sobre a Perfeição e a Realidade, Lisboa, Fim de Século, 2003, 152 pp.

37. LASAGA MEDINA, José - "Creencia y verdad en el contexto de la razón histórica”. In Jesús Díaz / Ma Carmen López Saénz (eds.) Fenomenología e Historia. Madrid: U.N.E.D., 2003, pp. 67-78.

38. LEDESMA, Felipe - "Heidegger y la Historia. Notas sobre la posibilidad de lo histórico". In Jesús Díaz / Ma Carmen López Saénz (eds.) Fenomenología e Historia. Madrid: U.N.E.D., 2003, pp. 49-59.

39. LÓPEZ SÁENZ, María del Carmen - "El sentido de la aisthesis en M. Merleau-Ponty". In José Manuel Santos, Pedro M. S. Alves, André Barata (eds.) - A Fenomenologia Hoje. Actas do primeiro Congresso da Associação Portuguesa de Filosofia Fenomenológica (Outubro de 2002). Lisboa: Centro de Filosofia da Universidade de Lisboa, 2003, pp. 299-310.

40. LÓPEZ SÁENZ, María del Carmen - "Feminismo y racionalidad ampliada", Contrastes, Málaga (2003) 93-107. 
41. LÓPEZ SÁENZ, María del Carmen / DIAZ ÁLVAREZ, Jesús. M. (eds.) - Fenomenología e Historia. Madrid: UNED, 2003.

42. LÓPEZ SÁENZ, María del Carmen - "Imaginación carnal en M. Merleau-Ponty", Revista de Filosofia, Madrid, 1 (2003) 157-169.

43. LÓPEZ SÁENZ, María del Carmen - "La determinación histórica de la hermenéutica de Hans-Georg Gadamer". In Jesús Díaz / Ma Carmen López Saénz (eds.) - Fenomenología e Historia. Madrid: U.N.E.D., 2003, pp. 167-181.

44. LÓPEZ SÁENZ, María del Carmen - "Reflexiones sobre la verdad de la filosofía hermenéutica de H. G.Gadamer", Cuadernos de filosofia Latinoamericana, vol. 88-89 (2003) 37-56.

45. MARTÍNEZ GUZMÁN, Vicent - "Historia y responsabilidad". In Jesús Díaz / Ma Carmen López Saénz (eds.) - Fenomenología e Historia. Madrid: U.N.E.D., 2003, pp. 217-226.

46. MATOS DIAS, Isabel - "Para uma ontologia do hábito. Leitura de De l'Habitude de Félix Ravaisson, Phainomenon, Lisboa, 5-6 (2002/ 2003), pp. 401-409.

47. MATOS DIAS, Isabel - "Telas e Teias. Vieira Tecedeira e a Poesia de Sophia", com Inês Barahona de Almeida, in As teias que as mulheres tecem, Maria Luisa Ribeiro Ferreira (dir.). Lisboa: Edições Colibri, 2003, pp. 39-72.

48. MATOS DIAS, Isabel - Cadernos de Viagem. José Pedro Croft, Santiago de Compostela: Xunta de Galicia, Centro Galego de Arte Contemporânea, 2003.

49. MORENO MÁRQUEZ, César - "El parecer clausurado. Notas sobre la apertura de la fenomenalidad y la ausencia de Logos". In $\mathrm{M}^{\mathrm{a}}$ del Carmen Paredes (ed $\mathrm{d}^{\mathrm{a}}$.) - Intencionalidad, mundo y sentido. Problemas de fenomenología y metafisica. Salamanca: Servicio de Publicaciones de la Universidad de Salamanca, 2003, pp. 143-160. 
50. NUNES, Etelvina Lopes - "Identidade e pessoalidade: um caminho e um processo". In: O que é ser humano? / What is it to be human? CD-Rom PC coordenação de João Carlos Major, Braga: Publicações da Faculdade de Filosofia - Universidade Católica Portuguesa, 2003.

51. PACHECO, Maria Adelaide - "A Presença de Heidegger em Portugal”, Revista Portuguesa de Filosofia, Braga, LIX/ 4 (2003), pp. 1203-1228.

52. PEREIRA, Miguel Baptista - "Alteridade, Linguagem e Globalização", Revista Filosófica de Coimbra, Coimbra, vol. XII, nº 23 (2003), pp. 3-38.

53. PEREIRA, Miguel Baptista - "A Hermenêutica da Condição Humana em Paul Ricoeur", Revista Filosófica de Coimbra, Coimbra, vol. XII, $\mathrm{n}^{\circ} 24$ (2003), pp. 235-278.

54. PEREÑA BLASI, Francesc - "Heidegger y la filosofía de la vida". In M. García-Baró / R. Pinilla, R. (eds.) - "Pensar la vida". Madrid: Servicio de Publicaciones de la Universidad Pontificia, 2003.

55. PEREÑA BLASI, Francesc - "Lebensphilosophie und wissenschaftliche Philosophie". In Lester Embree (ed.) - Issues Confronting the Post-european World, http://www.o-p-o.net (2003).

56. PICOT CASTRO, María Jesús - "La historia como escenario de las relaciones humanas en la filosofía de M. Merleau-Ponty". In Jesús Díaz / Ma Carmen López Saénz (eds.) - Fenomenología e Historia. Madrid: U.N.E.D., 2003, pp. 103-109.

57. PINTOS PEÑARANDA, María Luz - "Merleau-Ponty: fenomenología e historia". In Jesús Díaz / Ma Carmen López Saénz (eds.) Fenomenología e Historia. Madrid: U.N.E.D., 2003, pp. 87-102.

58. PINTOS PEÑARANDA, María Luz - "Coexistence empirique, apriori historique et projet de transformation de 1 ' histoire réelle. Trois perspectives phénoménologiques de la intersubjectivité". In Ion Copoeru / M. Diaconu / D. Popa (eds.) - Person, Community and Identity. Romania: House of the Book of Science, 2003, pp. 202-213. 
59. PINTOS PEÑARANDA, María Luz - "La gran aportación de la fenomenología husserliana para el mundo de hoy”. Escritos de Filosofia, Buenos Aires, 43 (2003) 125-156.

60. REIMÃO, Cassiano - "Problemática da Consciência em J.-P. Sartre: sua estrutura e implicações", Didaskalia, Lisboa, XXXIII/ 1-2 (2003), pp. 483-518.

61. REIS, José Encarnação - "O Tempo em Husserl”, Revista Filosófica de Coimbra, Coimbra, vol. XII, nº 24 (2003), pp. 399-490.

62. ROCHA, Acílio da Silva Estanqueiro - "A questão da 'universalidade da hermenêutica': controvérsias, aporias, pludimensionalidade” (II), Phainomenon, Lisboa, 7 (2003), pp. 53-86.

63. ROSA, José - "Da identidade narrativa. Paul Ricoeur, leitor do livro XI de Confissões, de Santo Agostinho", in Os longos caminhos do Ser. Homenagem a Manuel Barbosa da Costa Freitas, (Org. Cassiano Reimão; Coord. Manuel Cândido Pimentel). Lisboa: Universidade Católica Editora, 2003, pp. 557-597.

64. SÁ, Alexandre Franco de - "A política sobre a linha", Revista Portuguesa de Filosofia, Braga, LIX/ 4 (2003), pp. 1121-1152.

65. SÁEZ RUEDA, Luis - "Comprensión y Convalecencia. Por una crítica existencial del nihilismo patológico". In J. J. Acero Fernández / J. A. Nicolás / L. Sáez Rueda (eds.) - El legado de Gadamer. Granada: Universidad de Granada, 2004, pp. 423-438.

66. SÁEZ RUEDA, Luis - "Die Spannung zwischen existenzieller und reflexiver Pathologiekritik", Deutsche Zeitschrift für Philosophie, Berlin, vol. 51, 6 (2003) 903-1072.

67. SAN MARTÍN, Javier - "El último Husserl". Escritos de Filosofia, Buenos Aires, 43 (2003) 41-74. 
68. SAN MARTÍN, Javier - "Epoché und Selbstversenkung. Der Anfang der Philosophie" / "Epojé y ensimismamiento. El comienzo de la filosofía". In Lester Embree (ed.) - Issues Confronting the Post-european World, http://www.o-p-o.net (2003).

69. SAN MARTÍN, Javier - "Epojé y ensimismamiento. El comienzo de la filosofía". Phainomenon, Revista de Filosofia, Lisboa, 7 (2003) 13-22 .

70. SAN MARTÍN, Javier - "Fenomenologie tvaří v tvár 21. stoletî". In Ivan Blecha (ed.) Fenomenologie v pohybu, Olomouc: Univerzita Palackého Olomouc, 2003, pp. 229-240.

71. SEGURA PERAITA, Carmen - "Hans-Georg Gadamer en diálogo con Aristóteles: hermenéutica y filosofía práctica". In J. J. Acero Fernández / J. A. Nicolás / L. Sáez Rueda (eds.) - El legado de Gadamer. Granada: Universidad de Granada, 2004, pp. 119-125.

72. SERRANO DE HARO, Agustín - "Acerca de la incierta desaparición del yo", Prólogo a la edición española de Jean-Paul Sartre La trascendencia del ego. Madrid: Síntesis, 2003, pp. 9-26.

73. SERRANO DE HARO, Agustín - "Hanna Arendt y la equivocidad de la historia". In Jesús Díaz / Ma Carmen López Saénz (eds.) - Fenomenología e Historia. Madrid: U.N.E.D., 2003, pp. 155-165.

74. SILVA, Rui Sampaio da - "Wittgenstein e a Hermenêutica", Phainomenon, Lisboa, 7 (2003), pp. 129-154.

75. SOARES, Maria Luísa Couto - "O juízo evidente. Notas sobre a teoria do juízo em Brentano", Phainomenon, Lisboa, 7 (2003), pp. 105$-128$.

76. SOARES, Maria Luísa Couto - "A Experiência de Si: Permanência e Identidade do Eu", in AA.VV., Sujeito e Passividade, org. de Adelino Cardoso e José M. de Miranda Justo, Lisboa, Colibri/ CFUL, 2003, pp. 125-128. 
77. TRILlES CALVO, Karina P. - "M. Merleau-Ponty: la insuficiencia de la ciencia y de la filosofía reflexiva para dar cuenta del mundo". Revista Laguna 12 (2003) 127-137.

78. TRILLES CALVO, Karina P. - "Merleau-Ponty: la alteridad en $\mathrm{Fe}$ nomenología de la percepción". Agora. Papeles de Filosofía 21/2 (2003) 155-173.

79. UMBELINO, Luís António - "A Natureza em Merleau-Ponty", Revista Portuguesa de Filosofia, LIX/ 3 (2003), pp. 713-742.

80. URABAYEN, Julia - "La posición en la existencia y la evasión del ser: las primeras reflexiones de Emmanuel Levinas”, Anuario Filosófico, Pamplona, vol.XXXVI 3 (2003) 743-774.

81. VILA-CHÃ, João - "A (im-)pertinência de Heidegger", Revista Portuguesa de Filosofia, Braga, LIX/ 4 (2003), pp. 963-980

82. VILA-CHÃ, João - "Dez anos de Estudos sobre Heidegger", Revista Portuguesa de Filosofia, Braga, LIX/ 4 (2003), pp. 1321-1366

83. ZURRO, María del Rosario - "Notas para releer la filosofía de J. P. Sartre". Convivium. Revista de Filosofia 16 (2003) 95-116.

\section{Traduções / Traducciones}

1. ARENDT, Hannah - Conferencias sobre la filosofia politica de Kant. Barcelona: Paidós Ibérica, 2003.

2. ARENDT, Hannah - Eichmann en Jerusalén. Barcelona: Lumen, 2003.

3. ARENDT, Hannah - Entre el pasado y el futuro: ocho ejercicios sobre la reflexión política. Barcelona: Península, 2003.

4. BRENTANO, Franz - "Textos acerca de la unidad plural de la conciencia y la conciencia interna". Revista de Filosofia, México, 108 
(2003) 7-31. Introducción, traducción y comentarios de Miguel García-Baró.

5. DERRIDA, Jacques - Papel máquina: la cinta de máquina de escribir y otras respuestas. Madrid: Trotta, 2003.

6. GADAMER, Hans Georg - Los caminos de Heidegger. Barcelona: Herder, 2003.

7. GURWITSCH, Aron - "El nihilismo de nuestro tiempo", Agora. Papeles de Filosofia, Santiago de Compostela, vol. 22, 2 (2003) 157-192. Traducción y comentarios de María Luz Pintos Peñaranda.

8. HEIDEGGER, Martin - O Conceito de Tempo, prólogo, trad. e notas de Irene Borges Duarte, Lisboa: Fim de Século, 2003, 96 pp.

9. HEIDEGGER, Martin - Camino de campo = Der Feldweg. Barcelona: Herder, 2003.

10. HEIDEGGER, Martin / JASPERS, Karl - Correspondencia (1920-1963). Madrid: Síntesis, 2003.

11. HEIDEGGER, Martin - Observaciones relativas al arte: la plástica, el espacio. Pamplona: Universidad Pública de Navarra, 2003.

12. HEIDEGGER, Martin - ¿Qué es metafisica?: seguido de "epílogo a ¿Qué es metafísica? e introducción a ¿Qué es metafísica? Madrid: Alianza Editorial, 2003. Traducción de Arturo Leyte y Helena Cortés.

13. HEIDEGGER, Martin - Ser y Tiempo. Madrid: Trotta, 2003.

14. HEIDEGGER, Martin - Tiempo y Ser. Madrid: Tecnos, 2003.

15. von HERRMANN, Friedrich-Wilhelm - "A Ideia de Fenomenologia em Heidegger e Husserl”, trad. de Pedro Sobral Pignatelli, revisão de Irene Borges-Duarte, Phainomenon, Lisboa, 7 (2003), pp. 155-196.

16. MERLEAU-PONTY, Maurice - Palestras, trad. de Artur Morão, Lisboa: Edições 70, 2003, 77 pp. 
17. RICOEUR, Paul - Critica y convicción. Madrid: Síntesis, 2003.

18. RICOEUR, Paul - Finitud y culpabilidad. Madrid: Trotta, 2003.

19. RICOEUR, Paul - La memoria, la historia, el olvido. Madrid: Trotta, 2003. Traducción de Agustín Neira.

20. SARTRE, Jean-Paul - Barioná, el hijo del trueno: un ateo que presenta mejor que nadie el misterio de la Navidad. Voz de Papel, 2003.

21. SARTRE, Jean-Paul - Goragalea. San Sebastián: Elkarlanean, 2003.

22. SARTRE, Jean-Paul - Gora galea. Irún: Alberdania, 2003.

23. SARTRE, Jean-Paul - La náusea. Barcelona: Editorial Sol 90, 2003.

24. SARTRE, Jean-Paul - La trascendencia del ego. Madrid: Síntesis, 2003.

25. SARTRE, Jean-Paul - O muro.Barcelona: Bibliotex Editor, 2003.

26. SCHELER, Max - A Concepção Filosófica do Mundo, trad. e posf. de João Tiago Proença, Porto: Porto Ed., 2003, 143 pp.

27. STEIN, Edith - Escritos esenciales. Santander: Sal Térrea, 2003.

28. STEIN, Edith - La estructura de la persona humana. Madrid: Biblioteca de Autores Cristianos, 2003. 


\section{4}

\section{Monografias e artigos / Monografías y artículos}

1. ALVES, Pedro M. S. - "Para uma fenomenologia da comunicação", in Actas do Colóquio Internacional - Heidegger, Linguagem e Tradução. Lisboa: Centro de Filosofia da Universidade de Lisboa, 2004, pp. 27-44.

2. ALVES, Pedro M. S. - “A Fenomenologia em Portugal”. In Actas do Encontro de Iberistas. Salamanca, 2004.

3. ANDLER, Daniel - "Contextualizando as crenças", in AA.VV., $O$ Processo da Crença, org. de Fernando Gil, Pierre Livet e João Pina Cabral. Lisboa: Gradiva, 2004, pp. 50-83.

4. BECH, Joseph Maria - "Introducción" a Martin Heidegger Ser y Tiempo. Barcelona: R.B.A.- Biblioteca de los Grandes Pensadores, 2004, pp. 11-33.

5. BERNARDO, Fernanda - "Como uma língua por inventar, a hospitalidade poética de Derrida”, Phainomenon, Lisboa, 9 (2004), pp. 9-67.

6. BLANC, Mafalda de Faria - "Intersubjectividade e Pessoa: uma reflexão sobre a estrutura comunitária do ser", Phainomenon, Lisboa, 9 (2004), pp. 135-150.

7. BLANC, Mafalda de Faria - "Arte de Habitar", Communio, Revista Internacional Católica, Lisboa, XXI/ 4 (2004), pp. 391-398.

8. BLANC, Mafalda de Faria - "Notícia sobre o $\mathrm{III}^{\circ}$ Congresso mundial de Fenomenologia”, Phainomenon, Lisboa, 8 (2004), pp. 227-228.

9. BLANC, Mafalda de Faria - "Artes e Letras: a Vocação mediadora da Filosofia", Revista da Faculdade de Letras, Lisboa, 28, 5 série (2004), pp. 191-205. 
10. BLANC, Mafalda de Faria - "Processualidade do Ser e Acto", Itinerarium, Lisboa, 180 (2004), pp. 435-462.

11. BORGES-DUARTE, Irene - Heidegger, Linguagem, Tradução. Actas do Colóquio Internacional. Março 2002. Lisboa: C.F.U.L., 2004, 550 págs. Edição coordenada por Irene Borges-Duarte, Fernanda Henriques e Isabel Matos Dias.

12. BORGES-DUARTE, Irene - "A tradução como fenomenologia: o caso Heidegger.” In: Heidegger, Linguagem, Tradução. Lisboa: C.F.U.L., 2004, pp. 445-457.

13. CALVO GONZÁLEZ, José Luis - "Jan Patocka y la Carta 77. Antropología fenomenológica crítica y activismo de los derechos humanos", Contrastes. Revista Internacional de Filosofia, Málaga, IX (2004) 41-58 .

14. CANTISTA, Maria José - Prefácio. Revista da Faculdade de Letras série de Filosofia (Actas do Colóquio "Olhares, 50 anos da morte de Teixeira de Pascoaes", FLUP, 7 a 9 de Abril de 2003) vol. XXI, $2^{\text {a }}$ série (2004), pp. 41-58

15. CHIRINOS, María Pía - "Aristóteles y el joven Heidegger: una aclaración sobre la influencia de Brentano". Anuario Filosófico XXXVII/2 (2004) 309-328.

16. CONILL, Jesús - "El sentido de la Noología". In J. A. Nicolás / O. Barroso (eds.) - Balance y perspectivas de la filosofia de X. Zubiri. Granada: Comares, 2004, pp. 117-128.

17. CONILL, Jesús - "Ética hermenéutica desde la razón experiencial gadameriana". In J.' J. Acero, J. A. Nicolás, J.A.P. Tapias (eds.) - El legado de Gadamer. Granada: Universidad de Granada, 2004, pp. 49-62 .

18. CONILL, Jesús - "Ortega y Zubiri”. In J. A. Nicolás / O. Barroso (eds.), Balance y perspectivas de la filosofia de X. Zubiri. Granada: Comares, 2004, pp. 483-497. 
19. CONILL, Jesús - "Analítica hermenéutica de la razón experiencial tras Nietzsche (Algunas virtualidades prácticas)". In XV Congreso Valenciano de Filosofia. Valencia, Valencia: Bancaja, 2004, pp. 121-130 .

20. CORREIA, João Carlos - "Um esboço da teoria da comunicação de Alfred Schütz", Phainomenon, Lisboa, 8 (2004), pp. 75-96.

21. CORREIA, João Carlos - "Elementos para uma teoria da comunicação: os contributos de Schutz e Luhmann para a 'construção social da realidade'”. In: José Manuel Santos e João Carlos Correia (org.), Teorias da Comunicação, colecção "Estudos de Comunicação". Covilhã: UBI, 2004.

22. CORREIA, João Carlos e SANTOS, José Manuel (edits.) - Teorias da Comunicação. Covilhã: UBI, 2004.

23. ESCOBAR, Sara - "logos apofántico y apertura al mundo. El carácter derivado de la verdad proposicional en Logik. Die Frage nach der Wahrheit”. Anuario Filosófico XXXVII/2 (2004) 361-390.

24. ESCRIBANO, Xavier - "Los signos del arte nos fuerzan a pensar. Arte y filosofía en diálogo con Merleau-Ponty, Proust y Deleuze”. In Taula. Quaderns de Pensament, Illes Balears, 38 (2004) 263-269.

25. ESCRIBANO, Xavier - Sujeto encarnado y expresión creadora. Una aproximación al pensamiento de Maurice Merleau-Ponty. Cabrils: Prom. Edicions, 2004.

26. FERNANDES, António Horta - "Ortega e o problema da verdade", Revista Portuguesa de Filosofia, LX/ 1 (2004), pp. 151-166.

27. FERNÁNDEZ BEITES, Pilar - "Conciencia y cuerpo: la posibilidad de un dualismo unitario", Revista de filosofia, México, 110 (2004) 37-75 .

28. FERNÁNDEZ BEITES, Pilar - "La densidad ontológica de lo psíquico: el subconsciente", Cuadernos salmantinos de filosofia, Salamanca, vol. XXXI (2004) 335-362. 
29. FERRER SANTOS, Urbano - Edición del número monográfico Fenomenología y Ciencias humanas. Daimon. Revista de Filosofia, Murcia, vol. 32, 2 (2004).

30. FERRER SANTOS, Urbano - "Aportaciones de la Fenomenología a la Teoría contemporánea de la acción", Daimon. Revista de Filosofia, Murcia, vol. 32, 2 (2004) 131-144.

31. GARCÍA-BARÓ, Miguel - "La fenomenología de Husserl como condición de posibilidad de la noología de Zubiri”. In Diego Gracia (ed.) - Desde Zubiri. Granada: Comares, 2004, pp. 73-102.

32. GIL, Fernando - "Em guisa de posfácio: crer, confiar", in AA.VV., $O$ Processo da Crença, org. de Fernando Gil, Pierre Livet e João Pina Cabral, Lisboa, Gradiva, 2004, pp. 424-446.

33. GONÇALVES, Jorge de Almeida - "A Consciência ainda por explicar”, Cadernos de Filosofia, Lisboa, 15 (2004), pp. 69-84.

34. HIGUERO, Francisco Javier - "La reconfiguración del mundo del texto en la propuesta hermenéutica de Paul Ricoeur". Convivium. Revista de Filosofia 17 (2004) 149-166.

35. HELENO, José Manuel - Engano e Fingimento. Lisboa: Fim de Século Edições, 2004.

36. HELENO, José Manuel - Recensão a "Teoria da Interpretação de Paul Ricoeur", de João Amaral Ribeiro, Phainomenon, Lisboa, 8 (2004), pp. 225-226.

37. HELENO, José Manuel - "Husserl: Tempo e Ego", Phainomenon, Lisboa, 8 (2004), pp. 31-54.

38. LARANJEIRA, Paulo Jorge de Sousa - A concepção narrativa da identidade pessoal em Paul Ricoeur (TM), Braga, UCP, 2004, 215 pp.

39. LÓPEZ SÁENZ, María del Carmen - "Apuntes antropológicos basados en una relación: M. Merleau-Ponty y J-P. Sartre”. In J. F. Sellés 
(ed.) - Modelos antropológicos del siglo XX. Pamplona: Servicio de Publicaciones de la Universidad de Navarra, 2004, pp. 83-115.

40. LÓPEZ SÁENZ, María del Carmen - "El cuerpo como razón y libertad encarnadas". In F. Birulés/ M. I. Peña (eds.) - La passió per la llibertat. A passion for freedom. Barcelona: Ediciones de la Universidad de Barcelona, 2004, pp. 179-186.

41. LÓPEZ SÁENZ, María del Carmen - "El cuerpo vivido como nudo de identidad y diferencias. Una alternativa al cuerpo objetivado". In Jacinto Choza / María Luz Pintos (eds.) - Antropología y Ética ante los retos de la Biotecnología. Monográfico en Sevilla: Thémata. Revista de Filosofia, 2004, pp. 141-148.

42. LÓPEZ SÁENZ, María del Carmen - "Interpretación feminista de la corporalidad: Merleau-Ponty revisitado”, Estudios filosóficos, 152 (2004) 45-58.

43. LÓPEZ SÁENZ, María del Carmen - "Intersubjetividad como intercorporeidad”, La lámpara de Diógenes, vol. 5 (2004)57-71.

44. LÓPEZ SÁENZ, María del Carmen - "La continuidad del ecofeminismo con la filosofía de M. Merleau-Ponty". In L. Cavana / A. H. Puleo / C. Segura (eds.) - Mujeres y ecología. Historia, Pensamiento y sociedad. Madrid: Almudayna, 2004, pp. 213-225.

45. LÓPEZ SÁENZ, María del Carmen - "Pensar el arte y la aisthesis desde una fenomenología encarnada". In P. Blasco / E. Burgos / J. Solana (eds.) - Las raíces de la cultura europea. Zaragoza: PUZ, 2004, pp. 547-564.

46. LÓPEZ SÁENZ, María del Carmen - "Reconocimiento y crítica de la tradición en H-G. Gadamer”. In J. J. Acero / J. A. Nicolás / J. A. Pérez Tapias (eds.) - El legado de Gadamer. Granada: Servicio de Publicaciones de la Universidad de Granada, 2004, pp. 349-361.

47. MARTINS, Maria Manuela Brito - "As Figuras da Intersubjectividade em Husserl”, Phainomenon, Lisboa, 9 (2004), pp. 151-173. 
48. MATOS DIAS, Isabel - Heidegger, Linguagem, Tradução. Actas do Colóquio Internacional. Março 2002. Organização e Introdução com Irene Borges-Duarte e Fernanda Henriques. Lisboa: Centro de Filosofia da Universidade de Lisboa, 2004.

49. MATOS DIAS, Isabel - "Tradução: Palimpsestos e Metamorfoses", in Heidegger, Linguagem, Tradução. Lisboa: Centro de Filosofia da Universidade de Lisboa, 2004, pp. 97-105.

50. MESSIAS, Jorge - "Ciência e Religião", Vértice, Lisboa, 115 (2004), pp. 97-101.

51. MORENO MÁRQUEZ, César - "El otro máquina”. In J. V. Arregui / P. Martínez Freire / J. García González (eds.) - Cuerpos, subjetividades y artefactos, Granada: Repografía Digital, 2004.

52. MORENO MÁRQUEZ, César - "Break / Freak: fenómeno. (Notas para una geneidética: eidos y monstrum)”. Daimon. Revista de Filosofia, Murcia, 32 (2004) 55-75.

53. MORENO MÁRQUEZ, César - "La actitud virtual. Reflexiones sobre la alteridad intencional y el efecto-extrañamiento", Phainomenon. Revista de Fenomenologia, Lisboa 8 (2004) 97-117.

54. MORENO MÁRQUEZ, César - "Rostros sin Mundos. Inmanencia de la proximidad y pathos de lo interhumano en la metafísica de E. Lévinas". In Moisés Barroso / David Pérez Chico (eds.) - Un libro de huellas. Aproximaciones al pensamiento de Emmanuel Lévinas. Madrid: Trotta, 2004, pp. 149-176.

55. MORENO MÁRQUEZ, César - "Vértigo de la posibilidad, complejidad y axiología", Escritos de Filosofia, Buenos Aires, 44 (2004) 139-162 .

56. NUNES, Etelvina Lopes - "Identidade e pessoalidade: um caminho e um processo". Em: Consciência e Cognição DINIS, A. e CURADO, J. M. (Org.) Braga: Publicações da Faculdade da Filosofia de Braga, 2004, pp. 97-107. 
57. ORTEGA Y GASSET, José - Introducción a una estimativa. ¿Qué son los valores? Madrid: Encuentro Ediciones, 2004.

58. PEREIRA, Miguel Baptista - "Para uma Filosofia do Símbolo", Revista Filosófica de Coimbra, Coimbra, vol. XIII, n 25, (2004), pp. 3-30.

59. PINTOS PEÑARANDA, $M^{\mathrm{a}} \mathrm{Luz}$ - "Fenomenología del objeto vivido frente al olvido del objeto-para-el-sujeto por parte del 'objetivismo' naturalista”. Duererías. Revista de Filosofia, Zamora, 4 (2004) 135-158 .

60. PINTOS PEÑARANDA, $M^{\mathrm{a}}$ Luz - “ ‘Espacializamos! Fenomenología del espacio vivido frente al 'objetivismo' naturalista”. Alfa. Revista de la Asociación Andaluza de Filosofia, Jaén, 15 (2004) 17-39.

61. PINTOS PEÑARANDA, $M^{a}$ Luz - "La Fenomenología y las ciencias humanas y bio-sociales. Su convergencia en un importante momento de cambio de paradigmas". Revista Philosophica, Valparaíso-Chile, 27 (2004) 215-245.

62. REIS, José Encarnação - "O Tempo em Husserl (II)", Revista Filosófica de Coimbra, Coimbra, vol. XIII, n² 25 (2004), pp. 141-270.

63. SÁEZ RUEDA, Luis / J. J. ACERO / J.J. NICOLÁS (eds.) - El legado de Gadamer, Granada: Universidad de Granada, 2004.

64. SAN MARTÍN, Javier - "Análisis fenomenológico de la deducción trascendental de Kant (fragmento)". Éndoxa 18 (2004) 95-120.

65. SAN MARTÍN, Javier - "Juego, arte y cultura". Duererias. Revista de Filosofia, Zamora, 4 (2004) 73-85.

66. SAN MARTÍN, Javier - "Husserl in Ortega. O kritiki kulture v fenomenologiji”. Phainomena. Revija Fenomenološkega Društva v Ljubljani, vol. XIII, 49-50 (2004) 39-54. 
67. SAN MARTÍN, Javier - "La razón histórica como respuesta al idealismo de la modernidad". Revista de Estudios Orteguianos, Madrid, 8-9 (2004) 287-295.

68. SANTOS, José Manuel - "A comunicação e a estranheza do mundo". In: José Manuel Santos e João Carlos Correia (org.), Teorias da Comunicação, colecção "Estudos de Comunicação". Covilhã: UBI, 2004.

69. SANTOS, José Manuel e CORREIA, João Carlos (edits.) - Teorias da Comunicação. Covilhã: UBI, 2004.

70. SERRA, Paulo - "Proximidade e comunicação". In: José Manuel Santos e João Carlos Correia (org.), Teorias da Comunicação, colecção "Estudos de Comunicação". Covilhã: UBI, 2004.

71. SIMÕES, Luís Miguel - "A crítica de Derrida à teoria da significação de Husserl. A voz e o fenómeno e a primeira investigação lógica”, Phainomenon, Lisboa, 9 (2004), pp. 69-114.

72. SOARES, Maria Luísa Couto - "Crença e racionalidade prática: aproximações no plural”, in AA.VV., O Processo da Crença, org. de Fernando Gil, Pierre Livet e João Pina Cabral, Lisboa, Gradiva, 2004, pp. 298-318.

73. TENDEIRO, Maria da Graça - "As relações virtuais: que pessoa?", Phainomenon, Lisboa, 8 (2004), pp. 133-156.

74. TEIXEIRA, Joaquim de Sousa - Ipseidade e Alteridade: uma leitura da obra de Paul Ricoeur, Lisboa: IN-CM, 2004, 2 vols.

75. TIRADO SAN JUÁN, Victor Manuel - "En torno al sujeto o cómo ubicar a Zubiri en la postmodernidad". In Juan A. Nicolás / Óscar Barroso - Xavier Zubiri: un pensamiento metafísico que arraiga en lo real. Granada: Comares, 2004, pp. 553-568.

76. TIRADO SAN JUÁN, Victor Manuel - "Libertad práctica y libertad estética”. Cuadernos Salmantinos de Filosofia, XXXI (2004) 317-334. 
77. TIRADO SAN JUÁN, Victor Manuel - “Zubiri y Husserl”. In Juan A. Nicolás / Óscar Barroso - Xavier Zubiri: un pensamiento metafísico que arraiga en lo real. Granada: Comares, 2004, pp. 401-418.

78. TRILLES CALVO, Karina P. - "El cuerpo doliente. Una aproximación fenomenológica”. In Enric Casaban (ed.) - Josep L. Blasco in memoriam, Valencia: Societat del País Valencià, 2004, pp. 307-315.

79. TRILlES CALVO, Karina P. - "El cuerpo vivido. Algunos apuntes desde Merleau-Ponty”. In Jacinto Choza / María Luz Pintos (eds.) Antropología y Ética ante los retos de la Biotecnología. Monográfico en Sevilla: Thémata. Revista de Filosofia, 2004, pp. 135-140.

80. TRILlES CALVO, Karina P. - "Psicología fenomenológica. Un análisis existencial de la alucinación desde Maurice Merleau-Ponty”. Daimon: Revista de Filosofia, 32 (2004) 117-130.

81. URABAYEN, Julia - "Abraham frente a Ulises. La crítica de Emmanuel Levinas a la filosofía occidental”, Estudios Filosóficos, Valladolid, vol. LIII 153 (2004) 313-331.

\section{Traduções / Traducciones}

1. ARENDT, Hannah - Canción del verano y otros poemas. Santander: Límite, 2004.

2. ARENDT, Hannah - La tradición oculta. Barcelona: Paidós Ibérica, 2004.

3. ARENDT, Hannah - Sobre la revolución. Madrid: Alianza Editorial, 2004.

4. ARENDT, Hannah - Una revisión de la historia judia y otros ensayos. Barcelona: Paidós Ibérica, 2004.

5. DERRIDA, Jacques - Acabados. Madrid: Trotta, 2004. 
6. DERRIDA, Jacques - Rodar palabras. Madrid: Arena Libros, 2004.

7. GADAMER, Hans Georg - Hermenéutica de la Modernidad: conversaciones con Silvio Vietta. Madrid: Trotta, 2004.

8. HEIDEGGER, Martin - Hinos de Hölderlin, trad. de Lumir Nahodil, Lisboa: Instituto Piaget, 2004, 283 pp.

9. HEIDEGGER, Martin - O que é a Metafisica, trad. e nota introdutória de Carlos Morujão, Lisboa: Relógio d'Água, 2004, 80 pp.

10. HEIDEGGER, Martin - Carta sobre el humanismo. Madrid: Alianza Editorial, 2004. Traducción de Arturo Leyte y Helena Cortés.

11. HEIDEGGER, Martin - Idazki hautatuak. Vizcaya: Klasikoak, 2004.

12. HEIDEGGER, Martin - Lógica. La pregunta por la verdad. Madrid: Alianza Editorial, 2004. Traducción de Joaquín A. Ciria Cosculluela.

13. HEIDEGGER, Martin - ¿Qué es filosofia? Barcelona: Herder, 2004.

14. HENRY, Michel - Palabras de Cristo. Salamanca: Sígueme, 2004.

15. LEVINÁS, Emmanuel - Dificil libertad. Madrid: Caparrós Editores, 2004.

16. LEVINÁS, Emmanuel - La teoría fenomenológica de la intuición. Salamanca: Sígueme, 2004.

17. MERLEAU-PONTY, Maurice - $O$ Olho e o Espírito, pref. de Claude Lefort, trad. de Luís Manuel Bernardo, Lisboa: Vega, 2004 (5ª), 74 $\mathrm{pp}$.

18. MERLEAU-PONTY, Maurice - "Prólogo" de Fenomenología de la Percepción. Contrastes. Revista Internacional de Filosofia, Málaga, IX (2004) 185-211. Traducción crítica, introducción y apéndice de Benito Arias García. 
19. PATOCKA, Jan - El movimiento de la existencia humana. Madrid: Encuentro, 2004. Traducción de Agustín Serrano de Haro / Teresa Padilla / Jesús María Ayuso. Prólogo y revisión de todos los textos a cargo de Agustín Serrano de Haro.

20. RICOEUR, Paul - "Estudos sobre as Meditações Cartesianas de Husserl”, trad. de João Amaral, Phainomenon, Lisboa, 9 (2004), pp. 215-243 .

21. RICOEUR, Paul - Edmund Husserl - "A quinta meditação cartesiana", trad. de João Amaral, Phainomenon, Lisboa, 9 (2004), pp. 245-270 .

22. RICOEUR, Paul - Finitud y culpabilidad. Madrid: Trotta, 2004. Traducción de Cristina de Peretti / Julio Díaz Galán / Carolina Meloni.

23. SARTRE, Jean-Paul - El existencialismo es un humanismo. Madrid: B. A. C., 2004.

24. SARTRE, Jean-Paul - Paisatge d'un segle. Lleida: Publicacions dels Paisos Catalans, 2004.

25. SCHÜTZ, Alfred - "Símbolo, Realidade e Sociedade", trad. de Joaquim Carlos Araújo, Pedro M.S. Alves e João Carlos Correia, revisão geral de Pedro M. S. Alves, Phainomenon, Lisboa, 8 (2004), pp. 157-223 .

26. STEIN, Edith - Sobre el problema de la empatía. Madrid: Trotta, 2004. Traducción de José Luis Caballero Bono. 